\title{
Test of unmanned surface vehicles to conduct remote focal follow studies of a marine predator
}

\author{
Carey E. Kuhn ${ }^{1, *}$, Alex De Robertis ${ }^{2}$, Jeremy Sterling ${ }^{1}$, Calvin W. Mordy ${ }^{3,4}$, \\ Christian Meinig ${ }^{4}$, Noah Lawrence-Slavas ${ }^{4}$, Edward Cokelet ${ }^{4}$, Mike Levine ${ }^{2}$, \\ Heather Tabisola ${ }^{3,4}$, Richard Jenkins ${ }^{5}$, David Peacock ${ }^{5}$, Danny Vo ${ }^{6}$ \footnotetext{
Seattle, Washington 98115, USA

${ }^{2}$ Resource Assessment and Conservation Engineering Division, Alaska Fisheries Science Center, NOAA, Seattle, Washington 98115, USA

${ }^{3}$ Joint Institute for the Study of the Atmosphere and Ocean, University of Washington, Seattle, Washington 98195, USA

${ }^{4}$ Pacific Marine Environmental Laboratory, NOAA, Seattle, Washington 98115, USA

${ }^{5}$ Saildrone Inc., Alameda, California 94501, USA

${ }^{6}$ Wildlife Computers Inc., Redmond, Washington 98052, USA
} \\ ${ }^{1}$ Marine Mammal Laboratory, Alaska Fisheries Science Center, National Oceanic and Atmospheric Administration (NOAA),
}

\begin{abstract}
We tested the feasibility of using Saildrone unmanned wind- and solar-powered surface vehicles to conduct remote focal follow studies of northern fur seals Callorhinus ursinus. Using Argos satellite and transmitted GPS locations, the Saildrones followed a fur seal while recording oceanographic conditions and mapping prey abundance and depth distribution using a scientific echosounder. The Saildrones successfully followed 6 fur seals over $2.4 \pm 0.2 \mathrm{~d}$ (mean $\pm \mathrm{SE}$ ) and $149.7 \pm 16.3 \mathrm{~km}$ of the foraging path. Median separation distance between the Saildrone and fur seal path was $0.65 \pm$ $0.1 \mathrm{~km}$ and average time separation was $9.9 \pm 1.4 \mathrm{~h}$, with minimum time separations ranging from 1.9$4.9 \mathrm{~h}$. Time and distance separation were a function of both animal behavior and study design. Our results show that Saildrones can approach satellite tracked marine predators from long distances and follow them over extended periods while collecting oceanographic and prey data. These successful focal follows demonstrate that unmanned surface vehicles are a valuable tool for collecting data on fine-scale relationships between marine predators, their prey, and the environment.
\end{abstract}

KEY WORDS: Northern fur seal · Saildrone $\cdot$ Walleye pollock $\cdot$ Bering Sea

${ }^{*}$ Corresponding author: carey.kuhn@noaa.gov

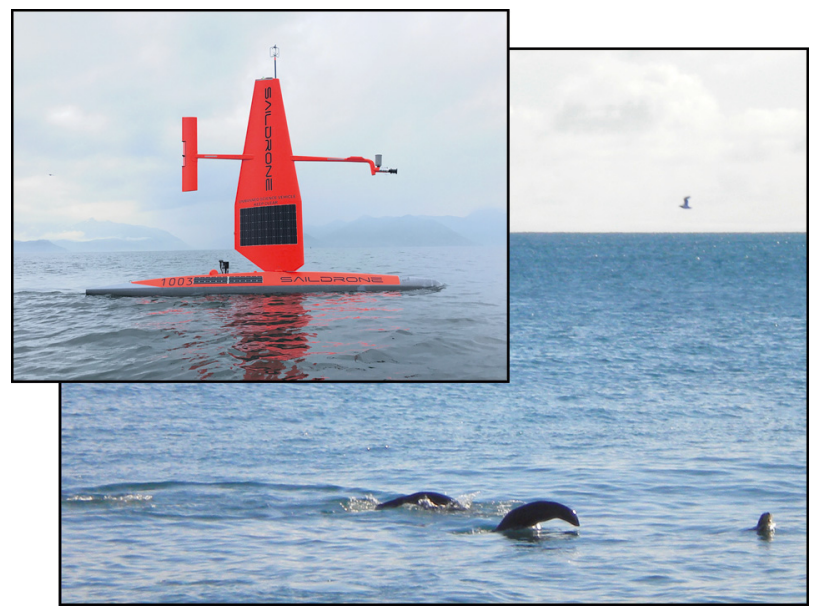

Unmanned surface vehicles (Saildrone) collected data on environmental conditions and prey availability while tracking northern fur seals over long distances and durations.

Photos: (Carey Kuhn, NOAA Fisheries (lower right), Saildrone Inc. (upper left))

\section{INTRODUCTION}

Focal follow studies can be used to gain detailed information about fine-scale relationships between marine predators, their habitat, and their prey (e.g. Friedlaender et al. 2015, Witteveen et al. 2015). Often

() C. Mordy, H. Tabisola, R. Jenkins, D. Peacock, D. Vo, and outside the USA the US Government 2020. Open Access under Creative Commons by Attribution Licence. Use, distribution and reproduction are unrestricted. Authors and original publication must be credited.

Publisher: Inter-Research · www.int-res.com 
vessel-based, focal follow studies record the behavior of a target animal either visually or by using biologging tools (e.g. dive recorders, video cameras, acoustic instruments) while simultaneously measuring physical and biological parameters at close distances $(<1 \mathrm{~km})$. Measurements of oceanographic parameters can be used to describe habitat, and echosounders or trawls can be used to quantify prey availability around the target animal (Friedlaender et al. 2015, Witteveen et al. 2015). These vessel-based studies are often costly and can be constrained by weather, day light, and location (NRC 2009, Verfuss et al. 2019), limiting the temporal and spatial scales that can be covered.

Unmanned vehicles, including underwater gliders and surface vehicles, are increasingly being used to supplement vessel-based studies (e.g. Cokelet et al. 2015, Rudnick 2016). These technologies can help expand research activities over longer time periods or larger areas, including shallow coastal regions inaccessible by large research vessels (Cokelet et al. 2015, Rudnick 2016). Recently, autonomous underwater vehicles (AUVs) have been used for focal follow type studies of fish, sharks, and turtles (Packard et al. 2013, Skomal et al. 2015, Dodge et al. 2018). These studies, which used acoustic transponders on the targeted animal, were temporally limited (1 to $<8 \mathrm{~h}$ ) and only able to measure oceanographic parameters, not prey abundance (Packard et al. 2013, Skomal et al. 2015, Dodge et al. 2018). For focal follow studies with unmanned vehicles to be comparable to traditional survey methods, longer follow durations and direct measurements of prey are needed.

Recently, unmanned wind- and solar-powered surface vehicles (USVs), called Saildrones (Saildrone Inc.), have successfully demonstrated their ability to conduct long-duration missions (>100 d), collecting data on oceanographic and atmospheric conditions and mapping fish depth distribution and abundance using a scientific echosounder (Cokelet et al. 2015, Mordy et al. 2017, De Robertis et al. 2019). We tested the feasibility of using Saildrones to conduct remote focal follow studies of northern fur seals Callorhinus ursinus over long distances (>100 km) and multiple days, to gain information about relationships between northern fur seals and their prey. We used near real-time locations transmitted from tracked fur seals to guide the Saildrones along a foraging path while collecting oceanographic and echosounder data. Here, we (1) demonstrate a successful feasibility test including performance metrics of the focal follows, (2) present examples of the data collected during the focal follows, and (3) provide suggestions for future improvement.

\section{MATERIALS AND METHODS}

\subsection{Fur seal instrumentation}

We equipped adult female northern fur seals on St. Paul Island, Alaska (see Fig. 1) with tracking instruments from July-October 2016 ( $\mathrm{n}=30$ ) and from July-September 2017 ( $n=16)$. A subset of the fur seals were selected as targets for the feasibility study. These fur seals were instrumented with either a SPLASH10 ( $\mathrm{n}=4 ;$ 2017) or SPLASH10-F ( $\mathrm{n}=2 ;$ 2016) glued to the pelage (Wildlife Computers [WC]). Both instrument types provided satellite locations via the Argos satellite system (hereafter 'satellite' locations) and sampled depth at $1 \mathrm{~s}$ intervals. SPLASH10-F instruments (2016 only) also recorded GPS locations at $15 \mathrm{~min}$ intervals ('archived GPS') and transmitted the most recent GPS location ('transmitted GPS') stored in the archive. In 2016, only transmitted GPS locations were used during focal follows because errors associated with GPS locations are significantly smaller than satellite location errors (Costa et al. 2010). To conserve battery power during periods of reduced satellite coverage, transmissions were duty-cycled (2016: off from 11:00-11:59 h GMT; 2017: off from 0:00-01:59 and 10:00-11:59 h).

\subsection{Study design}

This feasibility study occurred during a largescale, ecosystem-based research mission to map walleye pollock Gadus chalcogrammus ('pollock') abundance and depth distribution in the fur seal's foraging range (Mordy et al. 2017, De Robertis et al. 2019). The aim was to follow an individual for $\sim 2 \mathrm{~d}$ and collect data on oceanographic conditions and prey availability along the foraging path. In 2016, follows were initiated when a fur seal was near 1 of 2 Saildrones performing the foraging habitat survey (Mordy et al. 2017), with the goal of starting $\sim 12 \mathrm{~h}$ behind to ensure fur seal locations were consistently available to direct the Saildrone. Given that Saildrone speed is determined by environmental conditions, we could not predict in advance how long it would take to catch up to the targeted fur seal. To reduce time separation during the follows, in 2017 a single Saildrone was stationed $\sim 50 \mathrm{~km}$ from St. Paul Island prior to the start of the focal follows. When the first targeted fur seal began a foraging trip, the Saildrone was tasked to initiate the follow with no preset time delay. After a focal 
follow was completed, the Saildrone transited to the next closest fur seal to begin a new follow.

\subsection{Saildrone location acquisition and data collection}

Location data (satellite and transmitted GPS) were collected and stored in the WC Data Portal, and transmitted GPS data were processed on arrival. Although the standard procedure was for the portal to acquire new data from Argos at 15 min intervals, intermittent monitoring found that in 2017, the time between queries ranged from $~ 15$ min to slightly over $1 \mathrm{~h}$. When a delay was identified, new data were manually downloaded from the Argos website and uploaded into the WC portal until the time delay was reduced.

Saildrone Inc. acquired the location data in an automated fashion via WC's web services at $5 \mathrm{~min}$ intervals. The location data were filtered to remove erroneous locations (details in Text S1 in the Supplement at www.int-res.com/articles/suppl/m635p001_ supp.pdf). When new locations were received, an alert was sent to a Saildrone operator and the Saildrone was rerouted as necessary, generally within 5-10 min. When the Saildrone reached the last available location, it moved between the 2 final locations (2016) or stayed on-station (2017) until a new fur seal location became available.

During the Saildrone deployments, 19 (2016) and 17 (2017) oceanographic and atmospheric parameters were measured (see Supplement). Echosounder data, used to measure fish depth distribution and abundance, were processed using methods developed specifically for Saildrone data (De Robertis et al. 2019). In the study region, acoustic backscatter is dominated by scattering from pollock (De Robertis et al. 2019), the northern fur seal's primary prey when foraging on the Bering Shelf (Zeppelin \& Ream 2006). Acoustic-trawl surveys conducted from a research vessel in July 2016 confirmed that acoustic backscatter could be primarily attributed to pollock (De Robertis et al. 2019).

\subsection{Performance metrics}

Fur seal locations were filtered using a maximum transit rate of $3 \mathrm{~m} \mathrm{~s}^{-1}$ and hourly locations were modeled using a continuous-time correlated random walk (R v.3.6.0, packages 'argosfilter' and 'crawl'; Johnson et al. 2008; www.r-project.org). Modeled location data, instead of linear interpolated data, were used to determine the Saildrones' performance metrics because they more accurately reflect marine predator movement and can account for location errors (Tremblay et al. 2006, Johnson et al. 2008).

To quantify the focal follow performance, we calculated the distance separation between the modeled fur seal path and the Saildrone sampling path using straight-line distance to the closest point. Time separation was calculated as the time it took the Saildrone to reach points at $10 \mathrm{~km}$ intervals along the fur seal path. Time and distance separation were also calculated at the start and end of the fur seal path. Additionally, distance separation was calculated at the initiation of the focal follow, meaning the time at which the Saildrone was rerouted to move towards the start of the fur seal's path. Gap times in fur seal locations were calculated as the time between transmitted locations after applying the Saildrone filtering criteria. Results are summarized as means \pm SE. Differences between grouped summary metrics were tested using $t$-tests after validating normality and homogeneity of variances. The relationship between location gap times and distance separation was tested using a linear regression after log transformation of the data.

\section{RESULTS}

A total of 6 focal follows were conducted, sampling $149.7 \pm 16.3 \mathrm{~km}$ of the fur seals' foraging path (Table 1, Fig. 1). The fur seals covered this distance in $2.4 \pm 0.2 \mathrm{~d}$, whereas the Saildrone sampled the same path in $2.0 \pm 0.2 \mathrm{~d}$ (Table 1, and see the Supplement). Locations per hour were $47 \%$ higher when using satellite locations (2017) compared to transmitted GPS locations (2016, $t_{4}=3.17, \mathrm{p}=0.03$; Fig. S1A). Median gap times between transmitted locations were less than $1.5 \mathrm{~h}$ (Table 1), and maximum gap times (range: $4.8-9.9 \mathrm{~h}$ ) were significantly shorter in $2016\left(t_{4}=3.77, \mathrm{p}=0.02\right.$; Table 1, Fig. S1A).

The separation distances between the fur seal and the Saildrone at the initiation of the focal follows were lowest when focal follows were conducted opportunistically (2016) and when the Saildrone was near the rookery waiting to begin (NFSF0517; Table 1). In 2017, the separation distance at the initiation of the focal follows increased as the study progressed as fewer fur seals were available to be targets. Once the Saildrone reached the start of the focal follow path, median distance separations were $0.65 \pm 0.1 \mathrm{~km}$ (Table 1, Fig. S1B), with minimum sep- 


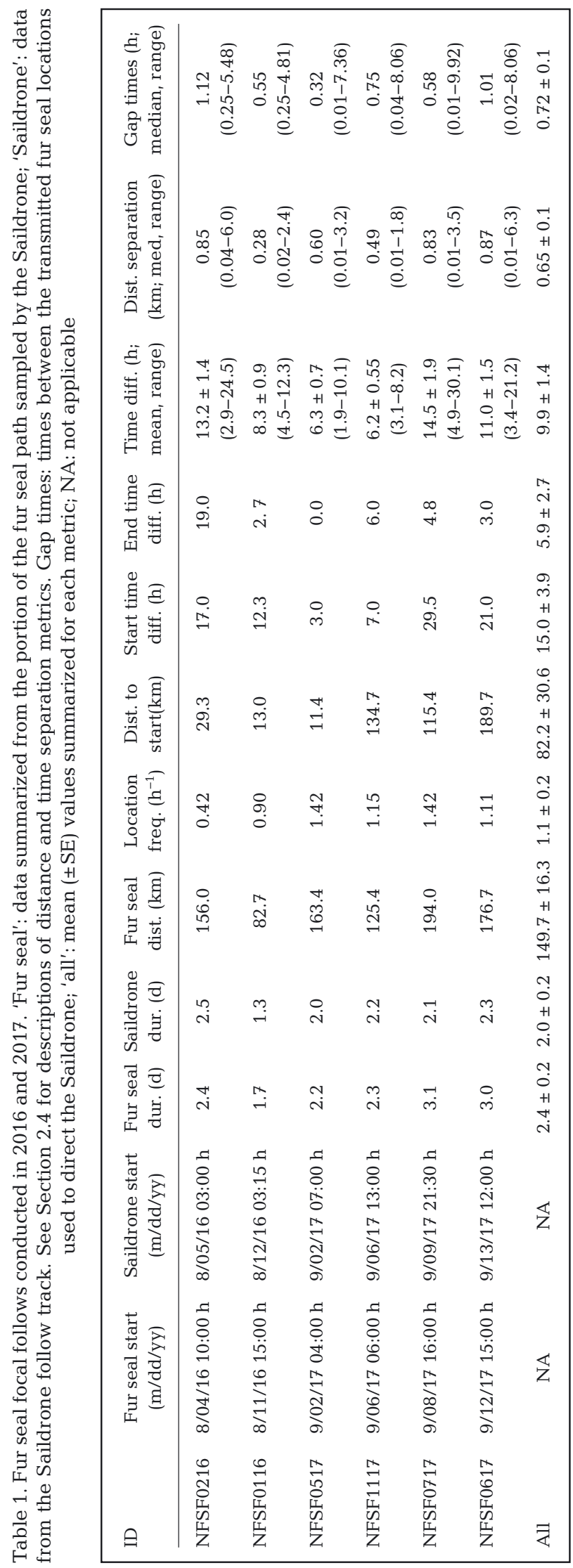

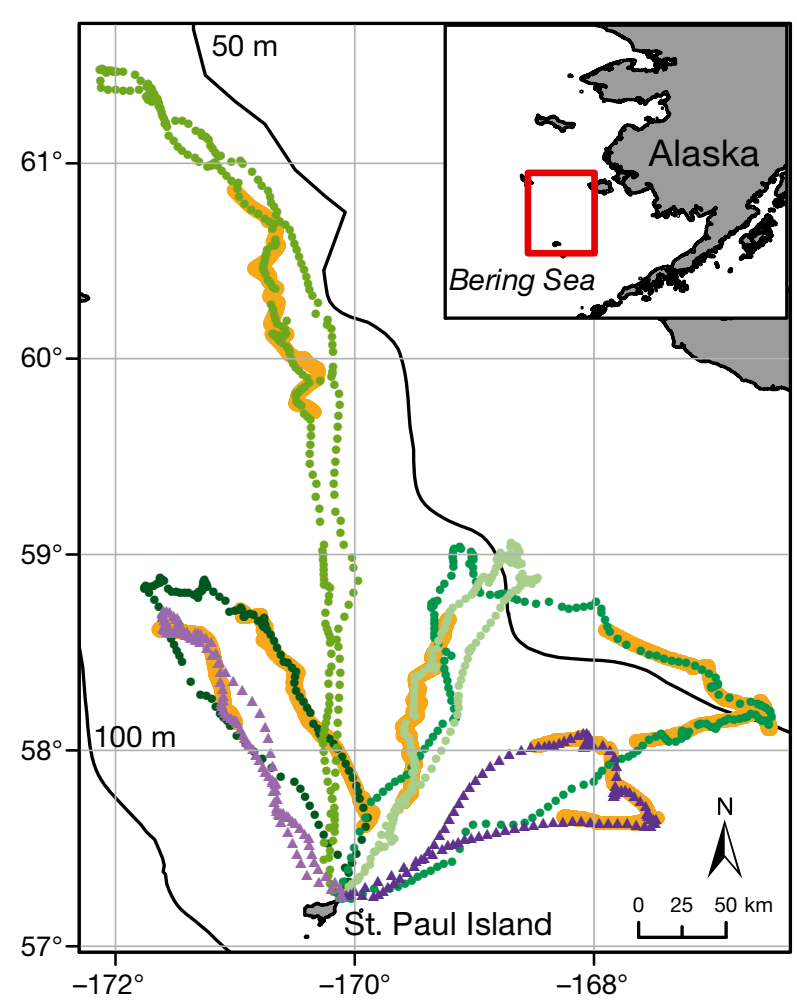

Fig. 1. Modeled hourly locations for 6 tracked northern fur seals (purple triangles: 2016; green points: 2017) with Saildrone follow paths overlaid in solid orange. Fur seals were instrumented on St. Paul Island, Alaska

aration distances averaging less than $15 \mathrm{~m}$ (Table 1, Fig. S1B). Separation distance was not related to mean location frequency, but increased when the time to the previous transmitted fur seal locations increased $\left(F_{1,353}=17.12, \mathrm{p}<0.001\right)$, likely due to the increasing uncertainty in the real-time location of the fur seal.

The average time separation between the Saildrone and the fur seal at the start of the focal follow was $15.0 \pm 3.9 \mathrm{~h}$, with the longest separations occurring at the start of the final 2 follows in 2017 (Table 1). As a focal follow progressed, average time separation decreased, with minimum values ranging from 1.9-4.9 h (Table 1, Fig. S1B). In all but one case, the Saildrone was able to significantly reduce the time separation from the start to the end of the focal follow period $\left(t_{5}=-2.11, \mathrm{p}=0.04\right.$; Table 1$)$.

Echosounder data were collected for each focal follow period, and oceanographic data were collected for all focal follows except the second follow in 2016 (NFSF0116) when it became necessary to conserve power. Example data are presented to demonstrate how this type of fine-scale data can ultimately be used to examine the influence of prey distribution 


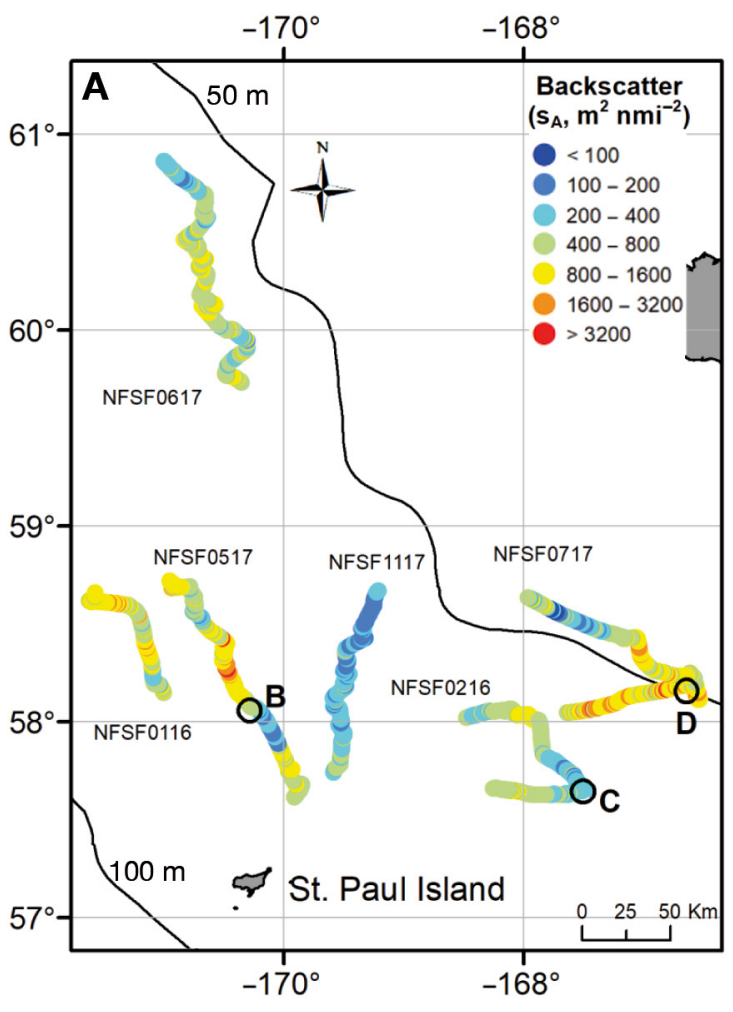

Fig. 2. (A) Total backscatter $\left(\mathrm{s}_{\mathrm{A}}\right)$ recorded by the Saildrone during northern fur seal focal follows. Black circles: location of the Saildrone for each dive plot. (B-D) Examples of backscatter with northern fur seal dive behavior overlaid (black line): (B) deep daytime dives, near the sea floor; (C) short, shallow dives occurring at night; (D) mixed shallow and deep dives at night. Echosounder data are presented from the nearest location (displaced by $5-7 \mathrm{~km}$ ) that matched the time of day the fur seal was diving (day vs. night). In (B) and (C), dive and echosounder data were collected simultaneously. In (D) the closest location with night echosounder data was sampled $24 \mathrm{~h}$ prior

and abundance on predator behavior (Fig. 2) and to demonstrate the fine-scale variability in oceanographic conditions along a foraging path (Fig. S2).

\section{DISCUSSION}

Our focal follow feasibility test showed that USVs, such as Saildrones, can remotely approach satellitetracked marine predators from long distances and follow them to collect valuable environmental and prey data over large distances and extended time periods. In a review of unmanned vehicles used for marine animal surveys, Verfuss et al. (2019) suggested powered vehicles are most suited for focal follow studies but acknowledged their limitations for
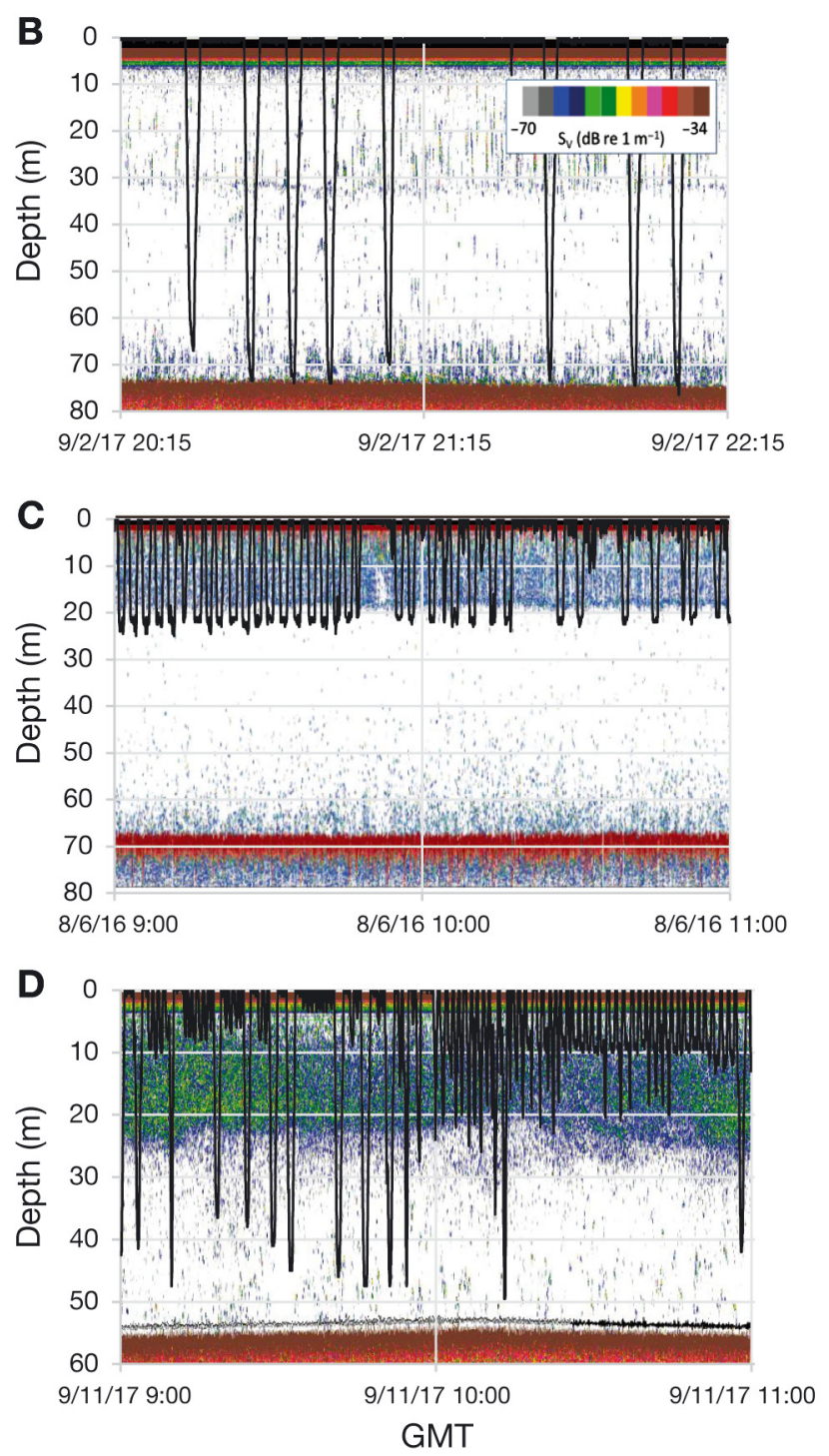

both duration and range. In contrast, Saildrones combine several key features for focal follow studies: relatively high speed, consistent communications to transmit data and effectively re-task the vehicle, the ability to make acoustic measurements of prey and measure environmental conditions, and long-term deployments (Cokelet et al. 2015, De Robertis et al. 2019).

The distance and time separations that are considered acceptable for focal follow research will vary for each study system based on factors including the distribution of prey, environmental features of interest (e.g. mesoscale vs. sub-mesoscale), and the variability of these features (Bost et al. 2009, Hazen et al. 2013). For northern fur seals, we were specifically interested in how the density and depth distribution 
of pollock influenced fur seal behavior. As spatial distributions of pollock are considered stable during the summer months (Smith \& Bakkala 1982) and layers of pollock can extend for 100+ km (Walline 2007), the performance metrics were acceptable for our research objectives. However, pollock depth distribution changes due to daily vertical migrations (Sogard \& Olla 1996). In order to account for these day/ night differences, in future analyses that relate fur seal dive behavior to the depth distribution of prey it will be necessary to match these data by time of day (day vs. night; e.g. Fig. 2). Matching by time of day may increase the time separation between the 2 data sources in some cases, but would not be required for examining other relationships, such as the influence of overall prey biomass or environmental characteristics.

\section{LIMITATIONS AND FUTURE APPLICATIONS}

Unlike traditional focal follow studies, our remote focal follow method introduced time delays throughout the data transfer process. Argos identifies 'mean disposal time' as the time between when an observation is collected by a platform (e.g. tracking instrument) and the time a user receives it (WMO 2015). Geographic location influences both mean disposal time $(<25 \mathrm{~min}$ to $1+\mathrm{h})$ and location frequency, as satellite pass frequency can be $4 \times$ higher in polar regions (WMO 2015, Jeanniard-du-Dot et al. 2017). This project included the added steps of having the WC Data Portal pull and process data from Argos before Saildrone Inc. acquired it to reroute the vehicle.

Several approaches could be used to improve focal follow performance by reducing the time or distance separation. Time separation could be reduced by increasing GPS acquisition frequency on the tracking instrument or by having Saildrone Inc. pull locations directly from Argos. Strategic positioning prior to a focal follow also reduced time separation, allowing the Saildrone to start the focal follow just $3 \mathrm{~h}$ behind the targeted fur seal. Future studies could incorporate other sources of data, such as acoustic tags similar to those used to track shark, fish, and turtles, to keep the Saildrone in close proximity (e.g. Packard et al. 2013, Skomal et al. 2015). Alternatively, the USV could potentially collect transmitted GPS locations directly from the tracking instrument, bypassing the Argos system (Jeanniard-du-Dot et al. 2017).

Being able to conduct focal follow studies remotely over longer distances and time periods opens up new possibilities for this type of fine-scale research, including studies of species in hard-to-reach or highly remote regions of the ocean. As the number of marine tracking studies continues to grow (Hussey et al. 2015), innovative technologies such as AUVs and USVs will be valuable tools to help understand the environmental and biological features that drive the behavior of marine species.

Acknowledgements. Northern fur seal research was conducted under Marine Mammal Protection Act permit \#14327 and the National Marine Fisheries Service (NMFS) IACUC A/NW 2013-3/2016-2. The research was supported by NOAA's Pacific Marine Environmental Laboratory (PMEL) and Alaska Fisheries Science Center, and by the University of Washington's Joint Institute for the Study of the Atmosphere and Ocean under NOAA Cooperative Agreement NA15OAR4320063. This is contribution 4958 to PMEL and Ecofoci-0926. Reference to trade names does not imply endorsement by NMFS/NOAA.

\section{LITERATURE CITED}

Bost CA, Cotté C, Bailleul F, Cherel Y and others (2009) The importance of oceanographic fronts to marine birds and mammals of the southern oceans. J Mar Syst 78:363-376

Cokelet ED, Meining C, Lawrence-Slavas N, Stabeno PJ and others (2015) The use of Saildrones to examine spring conditions in the Bering Sea: instrument comparisons, sea ice meltwater and Yukon River plume studies. Oceans 2015. IEEE, Washington, DC

Costa DP, Robinson PW, Arnould JPY, Harrison AL and others (2010) Accuracy of ARGOS locations of pinnipeds atsea estimated using Fastloc GPS. PLOS ONE 5:e8677

* De Robertis A, Lawrence-Slavas N, Jenkins R, Wangen I and others (2019) Long-term measurements of fish backscatter from unmanned surface vehicles and comparison with observations from a noise-reduced research vessel. ICES J Mar Sci 76:2459-2470

* Dodge KL, Kukulya AL, Burke E, Baumgartner MF (2018) TurtleCam: a 'smart' autonomous underwater vehicle for investigating behaviors and habitats of sea turtles. Front Mar Sci 5:90

Friedlaender AS, Goldbogen JA, Hazen EL, Calambokidis J, Southall BL (2015) Feeding performance by sympatric blue and fin whales exploiting a common prey resource. Mar Mamm Sci 31:345-354

*Hazen EL, Suryan RM, Santora JA, Bograd SJ, Watanuki Y, Wilson RP (2013) Scales and mechanisms of marine hotspot formation. Mar Ecol Prog Ser 487:177-183

*Hussey NE, Kessel ST, Aarestrup K, Cooke SJ and others (2015) Aquatic animal telemetry: a panoramic window into the underwater world. Science 348:1255642

Jeanniard-du-Dot T, Holland K, Schorr GS, Vo D (2017) Motes enhance data recovery from satellite-relayed biologgers and can facilitate collaborative research into marine habitat utilization. Anim Biotelem 5:17

Johnson DS, London JM, Lea MA, Durban JW (2008) Continuous-time correlated random walk model for animal telemetry data. Ecology 89:1208-1215

Mordy CW, Cokelet ED, De Robertis A, Jenkins R and others (2017) Advances in ecosystem research: Saildrone sur- 
veys of oceanography, fish, and marine mammals in the Bering Sea. Oceanography 30:113-115

NRC (National Research Council) (2009) Science at sea: meeting future oceanographic goals with a robust academic research fleet. The National Academies Press, Washington, DC

Packard GE, Kukulya A, Austin T, Dennett M and others (2013) Continuous autonomous tracking and imaging of white sharks and basking sharks using a REMUS-100 AUV. Oceans 2013. IEEE, San Diego, CA

Rudnick DL (2016) Ocean research enabled by underwater gliders. Annu Rev Mar Sci 8:519-541

Skomal GB, Hoyos-Padilla EM, Kukulya A, Stokey R (2015) Subsurface observations of white shark Carcharodon carcharias predatory behaviour using an autonomous underwater vehicle. J Fish Biol 87:1293-1312

Smith GB, Bakkala RG (1982) Demersal fish resources of the eastern Bering Sea: spring 1976. NOAA Tech Rep NMFS SSRF-754

Sogard SM, Olla BL (1996) Diel patterns of behavior in juvenile walleye pollock, Theragra chalcogramma. Environ Biol Fishes 47:379-386

Editorial responsibility: Elliott Hazen,

Pacific Grove, California, USA
Tremblay Y, Shaffer SA, Fowler SL, Kuhn CE and others (2006) Interpolation of animal tracking data in a fluid environment. J Exp Biol 209:128-140

*Verfuss UK, Aniceto AS, Harris DV, Gillespie D and others (2019) A review of unmanned vehicles for the detection and monitoring of marine fauna. Mar Pollut Bull 140: $17-29$

*Walline PD (2007) Geostatistical simulations of eastern Bering Sea walleye pollock spatial distributions, to estimate sampling precision. ICES J Mar Sci 64:559-569

*Witteveen BH, De Robertis A, Guo L, Wynne KM (2015) Using dive behavior and active acoustics to assess prey use and partitioning by fin and humpback whales near Kodiak Island, Alaska. Mar Mamm Sci 31:255-278

WMO (World Meteorological Organization) (2015) Report on Argos 2014-2015 operations and system improvements. www.argos-system.org/wp-content/uploads/2016/ 10/JTA-35-Doc-6-Argos-Operations-and-Improvements. pdf

Zeppelin TK, Ream RR (2006) Foraging habitats based on the diet of female northern fur seals (Callorhinus ursinus) on the Pribilof Islands, Alaska. J Zool 270:565-576

Submitted: June 12, 2019; Accepted: December 17, 2019

Proofs received from author(s): January 24, 2020 